\title{
Notícias sobre suicídio veiculadas em jornal brasileiro
}

\author{
News coverage on suicides published in Brazilian news media \\ channels
}

Renata da Silva Ferreira (https://orcid.org/0000-0001-5894-0116) ${ }^{1}$

Isabela dos Santos Martin (https://orcid.org/0000-0001-8274-4641) ${ }^{2}$

Ana Carolina Guidorizzi Zanetti (https://orcid.org/0000-0003-0011-4510) ${ }^{3}$

Kelly Graziani Giacchero Vedana (https://orcid.org/0000-0001-7363-2429) ${ }^{3}$
${ }^{1}$ Curso de Enfermagem, Escola de Enfermagem de Ribeirão Preto, Universidade de São Paulo (USP). R. Prof. Hélio Lourenço 3900, Vila Monte Alegre. 14040-902 Ribeirão Preto SP Brasil. renata.silva.ferreira@usp.br 2 Programa de Pós-

Graduação em Enfermagem Psiquiátrica, Escola de Enfermagem de Ribeirão Preto, USP. Ribeirão Preto SP Brasil.

${ }^{3}$ Departamento de Enfermagem Psiquiátrica e Ciências Humanas, Escola de Enfermagem de Ribeirão Preto, USP. Ribeirão Preto SP Brasil.

\begin{abstract}
This study analyzed how news coverage about suicides is published in the Brazilian electronic media and evaluated if the recommendations of the World Health Organization (WHO) were complied with in the online media. This quantitative, documentary and retrospective study investigated 89 published news items about suicide in 2017 in the Brazilian newspaper sites with the largest circulation. Descriptive statistics and tests of association were used for data analysis. The majority of news items contained neither content to be avoided nor aspects recommended by the WHO. The most frequent characteristics of the reported cases do not correspond directly to the epidemiological profile of suicidal behavior, but to media interests. Actions are needed to foster media involvement in suicide prevention, dissemination of resources and strategies to support and reduce stigma and the contagion effect.
\end{abstract}

Key words Suicide, Communications Media, Mental Health, Risk Factors, Brazil
Resumo O presente trabalho analisou como as notícias sobre suicídio são veiculadas em jornal eletrônico brasileiro e avaliou se as recomendações da Organização Mundial da Saúde (OMS) foram atendidas nessas publicações. Estudo quantitativo, documental e retrospectivo que investigou $89 \mathrm{no}$ tícias publicadas sobre suicídio no ano de 2017 no jornal brasileiro de maior circulação. Foi utilizada estatística descritiva e testes de associação para análise dos dados. A maioria das matérias não continha tanto aspectos contraindicados quanto aspectos recomendados pela OMS. As características mais frequentes dos casos noticiados não correspondem diretamente ao perfil epidemiológico do comportamento suicida, mas a interesses midiáticos. São necessárias ações favoreçam o envolvimento da mídia na prevenção do suicídio, divulgação de recursos e estratégias de apoio e redução de estigma e efeito contágio.

Palavras-chave Suicídio, Meios de Comunicação, Saúde Mental, Fatores de Risco, Brasil 


\section{Introdução}

O suicídio tem sido apontado como um sério e crescente problema de saúde pública mundial. Estima-se que a cada 40 segundos uma pessoa morre por suicídio. Os dados mundiais mostram que o suicídio é a segunda principal causa geral de morte entre pessoas de 15 a 29 anos, panorama que chama atenção por se tratar de uma situação considerada evitável em grande parte dos casos ${ }^{1}$. O Brasil é o oitavo país em números absolutos de suicídio no mundo; em 2012 ocorreram 11.821 óbitos decorrentes de lesões autoprovocadas voluntariamente, representando uma taxa de seis mortes a cada 100.000 habitantes no país $^{1}$. As taxas de tentativas de suicídio não letais são aproximadamente 20 vezes maiores do que as de suicídio e são um forte preditor de futura tentativa letal. Os números podem ser ainda maiores devido à subnotificação de casos e classificação errônea da causa de óbitos ${ }^{1,2}$.

O suicídio é um fenômeno complexo e multifatorial. É recomendável que não seja analisado em uma perspectiva unicausal ${ }^{1,2}$. Entre os múltiplos fatores envolvidos no risco de suicídio, a literatura científica aponta o contágio ou efeito Werther, um fenômeno de aparente disseminação de influências promotoras do comportamento suicida, no qual a mídia pode exercer importante papel $^{3}$. Esse fenômeno pode exercer importante impacto sobre pessoas vulneráveis ${ }^{3,4}$. Assim, é recomendável que a mídia seja envolvida nas ações de prevenção do suicídio para que possa colaborar com ações preventivas e evitar a disseminação de conteúdo pró-suicídio e o "efeito contágio"1,3.

A Organização Mundial da Saúde (OMS) apresenta recomendações explícitas sobre como notícias relacionadas ao suicídio devem ser veiculadas pela mídia ${ }^{5-7}$. São desejáveis as orientações sobre indicadores de risco e sinais de alerta ligados ao comportamento suicida, recursos de apoio, redução de estigma, alternativas para lidar com sofrimento, estressores de vida ou pensamentos suicidas, e como conseguir ajuda. Devese ter cautela com as notícias que envolvem celebridades ou entrevista de enlutados ${ }^{1,5-7}$.

Destaca-se ainda, a importância de que as notícias evitem abordagens simplistas, sensacionalistas, romantizadas, que promovam culpa, julgamento, enaltecimento, estereótipos, mitos ou a normalização do suicídio. Também são contraindicadas a divulgação da notícia em página de capa ou destaque, o uso de termos que relacionem o suicídio a ato "bem-sucedido", a divulgação de detalhes sobre nomes, métodos, local da ocorrência, bem como a exposição de imagens ou cartas suicidas ${ }^{1,5-7}$.

Considerando o exposto, este trabalho teve como objetivo analisar como as notícias sobre suicídio são veiculadas em jornal eletrônico brasileiro e avaliar se as recomendações da OMS foram atendidas nessas publicações.

\section{Método}

\section{Desenho e cenário do estudo}

Estudo documental, retrospectivo com abordagem quantitativa. Foram analisadas as notícias publicadas no jornal eletrônico com maior tiragem e circulação nacional no período da coleta de dados de acordo com o Instituto Verificador de Comunicação. Foi analisada a versão eletrônica do jornal devido à facilidade de acesso, propagação e interatividade com a população geral.

\section{Amostra}

Os critérios de inclusão utilizados foram todas as notícias publicadas no período de janeiro até dezembro de 2017 cujo conteúdo incluísse alguma palavra com o radical "suicid" empregado em sentido literal. Foram excluídas as notícias que utilizaram o termo "suicídio" apenas no sentido figurado, como "suicídio político e suicídio econômico" ( $\mathrm{n}=57)$, notícias com enfoque em outro assunto $(\mathrm{n}=216)$ e que abordavam o suicídio apenas em produções artísticas, como em livros, filmes e peças de teatro $(\mathrm{n}=77)$. No ano de 2017, 439 notícias continham o radical "suicid", após a aplicação dos critérios de exclusão, restaram 89 notícias que foram analisadas no presente estudo.

\section{Coleta de dados}

O jornal investigado disponibiliza acesso integral às notícias apenas para assinantes. Assim, foi realizada a assinatura do jornal para permitir o acesso integral a todas as publicações. O processo de coleta dos dados teve início com a busca das notícias. Na ferramenta de busca do site do jornal investigado, foi utilizado o radical "suicíd" para buscar por notícias que contivessem a palavra "suicídio" e derivações (suicida, suicidou, suicidaram, entre outras). Foi aplicado o filtro do site relacionado ao "período de publicação" para a busca de notícias publicadas no período de janeiro a dezembro de 2017. 
Todas as 439 notícias identificadas na busca foram lidas na íntegra para a seleção daquelas que seriam incluídas no estudo de acordo com critérios de seleção. As 89 notícias incluídas no estudo foram copiadas e posteriormente analisadas para a extração dos dados, que ocorreu por meio do preenchimento de um roteiro em formato digital elaborado pelas pesquisadoras com questões que permitiram a comparação das notícias publicadas com as recomendações da OMS ${ }^{1,5-7}$.

\section{Variáveis do estudo}

No presente estudo, foram analisadas variáveis sobre o caso noticiado: sexo da vítima, idade da vítima, quantidade de vítimas, desfecho (morte, lesão permanente, sem lesão permanente, não mencionado), localização geográfica, notícia referente a pessoas famosas (sim ou não), menciona pacto de suicídio (sim ou não) e menciona jogos de suicídio (sim ou não), caderno onde a notícia foi publicada.

Foram consideradas variáveis relacionadas à adequação das notícias segundo recomendações da OMS (com categorias de resposta sim ou não): menciona diagnóstico de transtorno mental, menciona sofrimento de pessoas próximas, informa como oferecer ajuda e contém informações sobre recursos de apoio. Também foi analisada a presença de elementos contraindicados pela OMS (com categorias de resposta sim ou não): apresenta nome da vítima, apresenta nome do familiar, local do evento, contém imagem da vítima, contém imagem da cena, menciona método para o suicídio, contém a expressão suicídio "bem-sucedido".

\section{Análise de dados}

Os dados coletados foram duplamente digitados em planilha no Programa Microsoft Excel 2010 e as planilhas foram comparadas para a correção de possíveis erros de digitação. Em segui$\mathrm{da}$, os dados digitados foram transportados para programa estatístico Statistical Package for Social Science (SPSS - versão 25), e analisados por estatística descritiva. Posteriormente, foi avaliada a normalidade da distribuição das medidas e, para avaliar as associações entre as variáveis do estudo, foram empregados os testes qui-quadrado e teste exato de Fisher, sendo adotado o nível de significância de 0,05 em ambos os testes.

\section{Aspectos éticos}

Os aspectos éticos desta pesquisa estão de acordo com a Resolução no 466, de 12 de dezembro de 2012, do Conselho Nacional de Saúde ${ }^{8}$.

\section{Resultados}

Neste estudo, foram analisadas 89 notícias relacionadas ao suicídio. A maior parte dos casos eram sobre uma única vítima $(71,9 \%)$, do sexo masculino $(60,7 \%)$ e sem idade relatada $(44,9 \%)$ e que foi a óbito $(57,3 \%)$. Não foram mencionados na maioria das notícias pactos $(94,4 \%)$ ou jogos suicidas $(97,8 \%)$, pessoas famosas $(57,3 \%)$, causas ou motivos do suicídio $(58,4 \%)$ depoimentos da vítima $(86,5 \%)$ ou de conhecidos $(85,4 \%)$.

A maior parte das publicações não relatava a localização geográfica do evento noticiado $(47,2 \%)$, mas os locais mais citados foram as cidades brasileiras São Paulo e Rio de Janeiro e o país norte-americano, Estados Unidos. A maioria das notícias $(59,5 \%)$ foi publicada em um dos seguintes cadernos "Cotidiano", "Ilustrada", "Colunas" e "Mundo".

A maioria das notícias não seguiu recomendações da OMS sobre mencionar diagnóstico de transtorno mental $(76,4 \%)$ e o sofrimento de pessoas próximas à vítima $(79,8 \%)$, informar como ajudar pessoas com comportamento suicida $(75,3 \%)$ e como acessar recursos de apoio (94,4\%) (Tabela 1).

A maioria das notícias não continha características contraindicadas pela OMS: utilizar a expressão suicídio "bem-sucedido" (98,9\%), expor nomes de locais onde o evento ocorreu (53,9\%), expor métodos de suicídio $(62,9 \%)$, nome de familiar $(85,4 \%)$, imagem da vítima $(92,1 \%)$ ou imagem da cena $(97,8 \%)$. O único aspecto contraindicado pela OMS presente na maioria das notícias foi a exposição do nome da vítima $(61,8 \%)$ (Tabela 2).

A divulgação de informações sobre "como oferecer ajuda a uma pessoa com comportamento suicida" (aspecto recomendado pela OMS) foi menor em notícias sobre uma única vítima, sobre vítimas do sexo feminino, adultas ou famosas e nos casos em que houve óbito ou ausência de lesão permanente (Tabela 3 ).

A exposição do nome da vítima (aspecto contraindicado pela OMS) foi mais frequente em notícias que envolviam pessoas famosas, com uma única vítima, do sexo feminino, adulta, e 


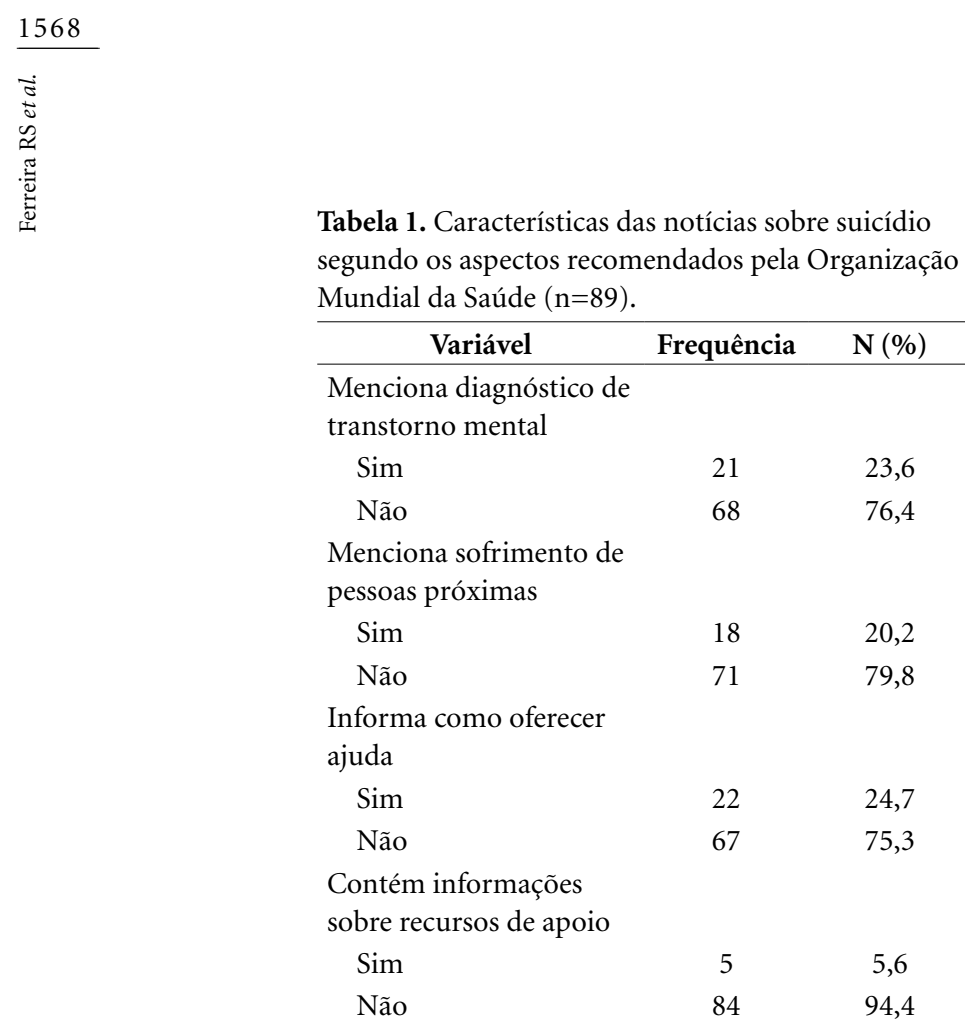

Tabela 2. Características das notícias sobre suicídio segundo as contraindicações da Organização Mundial da Saúde $(\mathrm{n}=89)$.

\begin{tabular}{|c|c|c|}
\hline Variável & Frequência & $\mathrm{N}(\%)$ \\
\hline \multicolumn{3}{|l|}{$\begin{array}{l}\text { Apresenta nome da } \\
\text { vítima }\end{array}$} \\
\hline Sim & 55 & 61,8 \\
\hline Não & 34 & 38,2 \\
\hline \multicolumn{3}{|l|}{ Local do evento } \\
\hline Residência & 13 & 14,6 \\
\hline Outros locais & 28 & 31,5 \\
\hline $\begin{array}{l}\text { Não se aplica/Não } \\
\text { mencionado }\end{array}$ & 48 & 53,9 \\
\hline \multicolumn{3}{|l|}{$\begin{array}{l}\text { Apresenta nome do } \\
\text { familiar }\end{array}$} \\
\hline Sim & 13 & 14,6 \\
\hline Não & 76 & 85,4 \\
\hline \multicolumn{3}{|l|}{$\begin{array}{l}\text { Contém imagem da } \\
\text { vítima }\end{array}$} \\
\hline $\operatorname{Sim}$ & 7 & 7,9 \\
\hline Não & 82 & 92,1 \\
\hline \multicolumn{3}{|c|}{ Contém imagem da cena } \\
\hline Sim & 2 & 2,2 \\
\hline Não & 87 & 97,8 \\
\hline \multicolumn{3}{|c|}{$\begin{array}{l}\text { Menciona método para o } \\
\text { suicídio }\end{array}$} \\
\hline Sim & 33 & 37,1 \\
\hline Não & 56 & 62,9 \\
\hline \multicolumn{3}{|c|}{$\begin{array}{l}\text { Menciona suicídio "bem- } \\
\text { sucedido" }\end{array}$} \\
\hline Sim & 1 & 1,1 \\
\hline Não & 88 & 98,9 \\
\hline
\end{tabular}

eventos ocorridos na residência da vítima e que resultaram lesão permanente (Tabela 4).

A exposição do método para o suicídio (aspecto contraindicado pela OMS) foi mais comum entre notícias sobre uma única vítima, do sexo masculino, adulta e em casos ocorridos na residência da vítima e que resultaram em óbito (Tabela 5).

Além das associações apresentadas anteriormente, foram testadas associações entre as características das notícias e cada uma das variáveis relacionadas a recomendações e contraindicações da OMS. No entanto, não foram identificadas associações estatisticamente significantes relacionadas às referidas variáveis e as demais características das notícias investigadas neste estudo.

\section{Discussão}

Esse é o primeiro estudo que se tem conhecimento realizado em contexto brasileiro sobre a adequação das notícias sobre suicídio veiculadas em jornal nacional, segundo as recomendações da OMS.

$\mathrm{Na}$ literatura alguns estudos revelaram inadequações na veiculação de notícias sobre suicídio no que se refere a presença de detalhes sobre o método utilizado, especulação indevida sobre os motivos do suicídio, não apresentação dos possíveis impactos na vida das famílias da vítima, estigma e sensacionalismo na elaboração da manchete e omissão de informações sobre transtornos mentais como fatores de risco para o suicídio ${ }^{9-11}$. Nossos resultados mostram que a maior parte das notícias não continha tanto os itens contraindicados quanto os recomendados pela OMS para abordagem do suicídio na mídia jornalística.

Destaca-se que uma parcela expressiva das notícias, $42,7 \%$, se referia a celebridades. Esse resultado representa mais os interesses da mídia do que o comportamento suicida, caso contrário, as pessoas famosas seriam um público altamente vulnerável ao comportamento suicida no Brasil. Os casos de suicídio entre famosos tendem receber maior atenção da mídia, o que pode ser prejudicial para pessoas vulneráveis ou que tenham admiração pela pessoa famosa mencionada na notícia ${ }^{4,12}$. A divulgação sobre o suicídio entre famosos pode potencializar os comportamentos imitativos ou de contágio. Estudos mostram que o suicídio de uma celebridade pode preceder um aumento significativo dos suicídios ${ }^{4,12}$.

A maioria das notícias era sobre casos com uma única vítima, do sexo masculino, sem idade 
Tabela 3. Associação entre as notícias que mencionavam como oferecer ajuda e características da vítima e do comportamento suicida e características da notícia $(\mathrm{n}=89)$.

\begin{tabular}{|c|c|c|c|}
\hline \multirow{3}{*}{ Variável } & \multicolumn{2}{|c|}{ Menciona como oferecer ajuda } & \multirow{3}{*}{ p-valor } \\
\hline & Sim & Não & \\
\hline & $\mathbf{N}(\%)$ & N (\%) & \\
\hline \multicolumn{4}{|l|}{ Local } \\
\hline Residência & $1(7,7)$ & $12(92,3)$ & 0,117 \\
\hline Outros & $5(17,9)$ & $23(82,1)$ & \\
\hline Não se aplica & $16(33,3)$ & $32(66,7)$ & \\
\hline \multicolumn{4}{|l|}{ Quantidade de vítimas } \\
\hline Uma vítima & $5(7,8)$ & $59(92,2)$ & $<0,001$ \\
\hline Mais de uma vítima & $3(37,5)$ & $5(62,5)$ & \\
\hline Não se aplica/não mencionado & $14(82,4)$ & $3(17,6)$ & \\
\hline \multicolumn{4}{|l|}{ Menciona pacto de suicídio } \\
\hline Sim & $2(40,0)$ & $3(60,0)$ & 0,594 \\
\hline Não & $20(23,8)$ & $64(76,2)$ & \\
\hline \multicolumn{4}{|l|}{ Menciona jogos de suicídio } \\
\hline Sim & $2(100,0)$ & $0(0,0)$ & 0,059 \\
\hline Não & $20(23,0)$ & $67(77,0)$ & \\
\hline \multicolumn{4}{|l|}{ Idade da vítima } \\
\hline Jovens (0 a 19) & $2(18,2)$ & $9(81,8)$ & 0,015 \\
\hline Adultos (20 a 59) & $2(6,7)$ & $28(93,3)$ & \\
\hline Idosos (Acima de 60) & $2(25,0)$ & $6(75,0)$ & \\
\hline Não informado & $16(40,0)$ & $24(60,0)$ & \\
\hline \multicolumn{4}{|l|}{ Sexo da vítima } \\
\hline Feminino & $0(0,0)$ & $14(100,0)$ & 0,001 \\
\hline Masculino & $8(14,8)$ & $46(85,2)$ & \\
\hline Não se aplica & $14(66,7)$ & $7(33,3)$ & \\
\hline \multicolumn{4}{|l|}{ Diagnóstico de transtorno mental } \\
\hline $\operatorname{Sim}$ & $4(19,0)$ & $17(81,0)$ & 0,575 \\
\hline Não & $18(26,5)$ & $50(73,5)$ & \\
\hline \multicolumn{4}{|l|}{ Desfecho } \\
\hline Morte & $6(11,8)$ & $45(88,2)$ & 0,001 \\
\hline Lesão permanente & $2(100,0)$ & $0(0,0)$ & \\
\hline Sem lesão permanente & $2(9,1)$ & $20(90,9)$ & \\
\hline Não mencionado/não se aplica & $12(85,7)$ & $2(14,3)$ & \\
\hline \multicolumn{4}{|l|}{ Menciona pessoas famosas } \\
\hline $\operatorname{Sim}$ & $5(13,2)$ & $33(86,8)$ & 0,046 \\
\hline Não & $17(33,3)$ & $34(66,7)$ & \\
\hline
\end{tabular}

citada, que resultaram em óbito. O comportamento suicida não letal é muito mais frequente do que o comportamento suicida letal ${ }^{1}$. Assim, o predomínio de casos que resultam em óbitos pode refletir as preferências e interesses da mídia por noticiar esses casos.

No presente estudo, as características dos casos noticiados parecem mais associados a interesses da mídia do que a dados epidemiológicos e a adesão a recomendações da OMS esteve associada a características dos casos noticiados.
É necessário que o envolvimento de profissionais de mídia nos assuntos relacionados ao suicídio não seja regido apenas por interesses midiáticos. Com isso, possibilita uma participação responsável desses agentes sociais na orientação da população e na divulgação dos meios de prevenção do suicídio, que possam promover resultados benéficos para a sociedade ${ }^{13,14}$.

No presente estudo, a maioria das notícias expos o nome da vítima, o que pode ter consequências negativas para a própria vítima (em casos 
Tabela 4. Associação entre expor o nome da vítima e características da notícia ( $\mathrm{n}=89$ ).

\begin{tabular}{|c|c|c|c|}
\hline \multirow{3}{*}{ Variável } & \multicolumn{2}{|c|}{ Nome da vítima } & \multirow{3}{*}{ p-valor } \\
\hline & Sim & Não & \\
\hline & $\mathrm{N}(\%)$ & $\mathrm{N}(\%)$ & \\
\hline \multicolumn{4}{|l|}{ Local } \\
\hline Residência & $12(92,3)$ & $1(7,7)$ & 0,012 \\
\hline Outros & $19(67,9)$ & $9(32,1)$ & \\
\hline Não se aplica & $24(50,0)$ & $24(50,0)$ & \\
\hline \multicolumn{4}{|l|}{ Quantidade de vítimas } \\
\hline Uma vítima & $51(79,7)$ & $13(20,3)$ & $<0,001$ \\
\hline Mais de uma vítima & $4(50,0)$ & $4(50,0)$ & \\
\hline Não se aplica/não mencionado & $0(0,0)$ & $17(100,0)$ & \\
\hline \multicolumn{4}{|l|}{ Menciona pacto de suicídio } \\
\hline Sim & $3(60,0)$ & $2(40,0)$ & 1,000 \\
\hline Não & $52(61,9)$ & $32(38,1)$ & \\
\hline \multicolumn{4}{|l|}{ Menciona jogos de suicídio } \\
\hline Sim & $0(0,0)$ & $2(100,0)$ & 0,143 \\
\hline Não & $55(63,2)$ & $32(36,8)$ & \\
\hline \multicolumn{4}{|l|}{ Idade da vítima } \\
\hline Jovens (0 a 19) & $8(72,7)$ & $3(27,3)$ & $<0,001$ \\
\hline Adultos (20 a 59) & $29(96,7)$ & $1(3,3)$ & \\
\hline Idosos (Acima de 60) & $6(75,0)$ & $2(25,0)$ & \\
\hline Não informado & $12(30,0)$ & $28(70,0)$ & \\
\hline \multicolumn{4}{|l|}{ Sexo da vítima } \\
\hline Feminino & $12(85,7)$ & $2(14,3)$ & $<0,001$ \\
\hline Masculino & $43(79,6)$ & $11(20,4)$ & \\
\hline Não se aplica & $0(0,0)$ & $21(100,0)$ & \\
\hline \multicolumn{4}{|l|}{ Diagnóstico de transtorno mental } \\
\hline Sim & $16(76,2)$ & $5(23,8)$ & 0,134 \\
\hline Não & $39(57,4)$ & $29(42,6)$ & \\
\hline \multicolumn{4}{|l|}{ Desfecho } \\
\hline Morte & $36(70,6)$ & $15(29,4)$ & $<0,001$ \\
\hline Lesão permanente & $2(100,0)$ & $0(0,0)$ & \\
\hline Sem lesão permanente & $16(72,7)$ & $6(27,3)$ & \\
\hline Não mencionado/não se aplica & $1(7,1)$ & $13(92,9)$ & \\
\hline \multicolumn{4}{|l|}{ Menciona a pessoas famosas } \\
\hline Sim & $34(89,5)$ & $4(10,5)$ & 0,000 \\
\hline Não & $21(41,2)$ & $30(58,8)$ & \\
\hline
\end{tabular}

não letais) ou para os familiares e outras pessoas próximas ${ }^{15}$. Em diferentes contextos sócioculturais $^{10,15,16}$, o estigma associado ao suicídio é expressivo e acarreta uma carga negativa para pessoas com comportamento suicida e familiares. Um estudo transversal realizado no Reino Unido apontou que indivíduos enlutados por suicídio têm níveis mais elevados de estigma percebido, vergonha, responsabilidade e culpa quando comparados com indivíduos enlutados por outros tipos de morte $^{17}$.
A exposição inadequada de casos de suicídio comumente tem o objetivo de alcançar maior repercussão da notícia e atrair a curiosidade do leitor $^{10}$. Assim, é importante que sejam desenvolvidas ações de sensibilização e formação dos profissionais da mídia em relação às especificidades da veiculação de notícias sobre suicídio.

A OMS aponta que aproximadamente 90\% das pessoas que morrem por suicídio tem algum tipo de transtorno mental ${ }^{1}$, contudo, a maioria das notícias investigadas no presente estudo não 
Tabela 5. Associação entre mencionar método para o suicídio e características da notícia $(\mathrm{n}=89)$.

\begin{tabular}{|c|c|c|c|}
\hline \multirow{3}{*}{ Variável } & \multicolumn{2}{|c|}{ Método do suicídio } & \multirow{3}{*}{ p-valor } \\
\hline & Sim & Não & \\
\hline & $\mathbf{N}(\%)$ & $\mathbf{N}(\%)$ & \\
\hline \multicolumn{4}{|l|}{ Local } \\
\hline Residência & $10(76,9)$ & $3(23,1)$ & $<0,001$ \\
\hline Outros & $14(50,0)$ & $14(50,0)$ & \\
\hline Não se aplica & $9(18,8)$ & $39(81,3)$ & \\
\hline \multicolumn{4}{|l|}{ Quantidade de vítimas } \\
\hline Uma vítima & $29(45,3)$ & $35(54,7)$ & 0,005 \\
\hline Mais de uma vítima & $3(37,5)$ & $5(62,5)$ & \\
\hline Não se aplica/não mencionado & $1(5,9)$ & $16(94,1)$ & \\
\hline \multicolumn{4}{|l|}{ Faz menção a pacto de suicídio } \\
\hline Sim & $3(60,0)$ & $2(40,0)$ & 0,355 \\
\hline Não & $30(35,7)$ & $54(64,3)$ & \\
\hline \multicolumn{4}{|l|}{ Faz menção a jogos de suicídio } \\
\hline Sim & $0(0,0)$ & $2(100,0)$ & 0,528 \\
\hline Não & $33(37,9)$ & $54(62,1)$ & \\
\hline \multicolumn{4}{|l|}{ Idade da vítima } \\
\hline Jovens ( 0 a 19$)$ & $5(45,5)$ & $6(54,5)$ & $<0,001$ \\
\hline Adultos (20 a 59) & $20(66,7)$ & $10(33,3)$ & \\
\hline Idosos (Acima de 60) & $4(50,0)$ & $4(50,0)$ & \\
\hline Não informado & $4(10,0)$ & $36(90,0)$ & \\
\hline \multicolumn{4}{|l|}{ Sexo da vítima } \\
\hline Feminino & $3(21,4)$ & $11(78,6)$ & $<0,001$ \\
\hline Masculino & $29(53,7)$ & $25(46,3)$ & \\
\hline Não se aplica & $1(4,8)$ & $20(95,2)$ & \\
\hline \multicolumn{4}{|l|}{ Diagnóstico de transtorno mental } \\
\hline Sim & $7(33,3)$ & $14(66,7)$ & 0,799 \\
\hline Não & $26(38,2)$ & $42(61,8)$ & \\
\hline \multicolumn{4}{|l|}{ Desfecho da vítima } \\
\hline Morte & $29(56,9)$ & $22(43,1)$ & 0,001 \\
\hline Lesão permanente & $0(0,0)$ & $2(100,0)$ & \\
\hline Sem lesão permanente & $4(18,2)$ & $18(81,8)$ & \\
\hline Não mencionado/não se aplica & $0(0,0)$ & $14(100,0)$ & \\
\hline \multicolumn{4}{|l|}{ Notícia se refere a pessoas famosas } \\
\hline Sim & $17(44,7)$ & $21(55,3)$ & 0,268 \\
\hline Não & $16(31,4)$ & $35(68,6)$ & \\
\hline
\end{tabular}

mencionava qualquer tipo de transtorno mental. A concepção do suicídio como uma questão que requer cuidados em saúde pode favorecer a busca por ajuda especializada, além de favorecer a redução do estigma ${ }^{18}$.

A maioria das notícias não informava como ajudar alguém com comportamento suicida ou informações sobre recursos de apoio. De acordo com a literatura, a divulgação de informações sobre apoio parece ser um aspecto menos abor- dado. Um estudo sobre a mídia jornalística canadense identificou, na maioria das notícias, a falta de informações sobre onde obter ajuda, apesar da elevada adesão às outras recomendações da $\mathrm{OMS}^{19}$. Pesquisas desenvolvidas em diferentes países sobre as reportagens relacionadas ao suicídio também revelam a carência de divulgação de informações sobre ações de prevenção do suicídio e locais de apoio ${ }^{9-11,20,21}$. Tais aspectos podem revelar a participação insuficiente da mídia na 
disseminação de informações úteis para a busca de assistência e busca por tratamento e de conteúdos que contribuam com a sociedade em geral.

A internet tem potencial para propiciar a busca por ajuda anônima, a superação de dificuldades de contato social, o estreitamento do vínculo com centros de ajuda ${ }^{22}$, a redução de estigma, a difusão de ações de promoção da saúde mental, apoio e prevenção do comportamento suicida $^{23,24}$. Os jornais on-line também poderiam favorecer o alcance desses benefícios.

A divulgação de diretrizes, a sensibilização e treinamento de profissionais da mídia são imperativos para que a mídia possa exercer uma ação segura e voltada para a prevenção. De acordo com relatório produzido pelo Institute for Mental Health in England, a maioria dos profissionais da mídia não possui treinamento e conhecimento suficiente sobre diretrizes que orientam divulgação de notícias de suicídio ${ }^{25}$.

A intensa comunicação por mídias e tecnologias digitais deve ser acompanhada de ações educativas em diferentes setores sociais para promover a conduta responsável e saudável relacionada à produção e divulgação de conteúdos digitais. Um trabalho desenvolvido na Austrália estimulou jovens do Ensino Médio a desenvolver mensagens para prevenção de suicídio veiculadas em mídias sociais. Os jovens avaliaram positivamente o programa e se perceberam mais capazes de oferecer apoio emocional on-line com segurança ${ }^{26}$. Outro exemplo da utilização de mídia digital para promoção de saúde mental e prevenção do suicídio são as campanhas que promovem difusão de informações e discussões em tempo real, um diferencial às intervenções de promoção da saúde mais tradicionais ${ }^{27}$. Assim, é importante investir na formação de profissionais da mídia, bem como na promoção de responsabilidade pessoal e participação social na produção e divulgação de conteúdos em ambientes digitais.

\section{Conclusão}

Este estudo analisou como as notícias sobre suicídio são veiculadas em jornal de maior circulação on-line no Brasil no período de um ano e comparou as características das notícias com as recomendações da OMS sobre o assunto.

Observou-se que as recomendações da OMS são parcialmente seguidas. Embora a maioria das matérias tenha evitado aspectos contraindicados, os aspectos desejáveis foram observados na minoria das notícias. As características mais frequentes dos casos noticiados não correspondem diretamente ao perfil epidemiológico do comportamento suicida, mas a interesses midiáticos, como os casos que envolvem óbitos, sexo masculino, pessoas famosas e sem transtorno mental divulgado.

Informações sobre como oferecer ajuda a uma pessoa com comportamento suicida foram menos frequentes em notícias sobre uma única vítima, do sexo feminino, adulta ou famosa e nos casos onde houve óbito ou ausência de lesão permanente. A exposição do nome da vítima esteve associada a casos envolvendo tentativas de suicídio não letais com lesão permanente, com uma única vítima, com mulheres, adultas, pessoas famosas e eventos ocorridos na residência da vítima enquanto a apresentação de método para o suicídio foi mais comum entre casos com uma única vítima, notícias sobre homens, adultos, eventos ocorridos na residência da vítima e que resultaram em óbito.

Os resultados apontam para a necessidade de reflexões, sensibilizações, monitoramento e ações educativas para promover o envolvimento seguro da mídia na divulgação de matérias relacionadas ao suicídio que colaborem com a prevenção do suicídio, redução de estigma e efeito contágio e conhecimento sobre recursos e estratégias de apoio. 


\section{Colaboradores}

KGG Vedana, colaborou na concepção, delineamento, análise e interpretação dos dados, redação do artigo e sua revisão crítica e aprovação da versão a ser publicada. IS Martin e ACG Zanetti colaboraram na redação do artigo, sua revisão crítica e aprovação da versão a ser publicada. RS Ferreira colaborou na concepção, delineamento, análise e interpretação dos dados.

\section{Referências}

1. World Health Organization (WHO). Preventing suicide. A global imperative. Washington: OPS; 2014.

2. Associação Brasileira de Psiquiatria (ABP). Suicídio: informando para prevenir. Brasília: CFM/ABP; 2014.

3. Niederkrotenthaler T, Voracek M, Herberth A, Till B, Strauss M, Etzersdorfer E, Eisenwort B, Sonneck G. Role of media reports in completed and prevented suicide: Werther v. Papageno effects. Br J Psychiatry 2010; 197:234-243.

4. Stack S. Media coverage as a risk factor in suicide. $J$ Epidemiol Community Health 2003; 57:238-240.

5. Organização Mundial da Saúde (OMS). Departamento de Saúde Mental, Transtornos Mentais e Comportamentais. Prevenção do Suicídio: um manual para profissionais da mídia. Genebra: OMS; 2000.

6. World Health Organization (WHO). Department of Mental Health and Substance Abuse. International Association for Suicide Prevention. Preventing suicide: a resource for media professionals. Genebra: WHO; 2008.

7. World Health Organization (WHO). Preventing suicide: a resource for media professionals, update 2017. Genebra: WHO; 2017.

8. Brasil. Conselho Nacional de Saúde (CNS). Resolução $n^{\circ}$ 466, de 12 dezembro de 2012. Trata de pesquisas em seres humanos e atualiza a Resolução 196. Diário Oficial da União 2012; $12 \mathrm{dez}$.

9. Chu X, Zhang X, Cheng P, Schwebel DC, Hu G. Assessing the use of media reporting recommendations by the World Health Organization in suicide news published in the most influential media sources in China, 2003-2015. Int J Environ Res Public Health 2018; $15: 451$.

10. Jain N, Kumar S. Is suicide reporting in Indian newspapers responsible? A study from Rajasthan. Asian J Psychiatr 2016; 24:135-138.

11. Utterson M, Daoud J, Dutta R. Online media reporting of suicides: analysis of adherence to existing guidelines. B J Psych Bull 2017; 41:83-86.

12. Jang SA, Sung JM, Park JY, Jeon WT. Copycat suicide induced by entertainment celebrity suicides in South Korea. Psychiatry Investig 2016; 13(1):74-81.

13. Cheng Q, Fu K, Caine E, Yip PSF. Why do we report suicides and how can we facilitate suicide prevention efforts? Perspectives of Hong Kong media professionals. Crisis 2014; 35(2):74-81.

14. Cheng Q, Chen F, Lee EST, Yip PSF. The role of media in preventing student suicides: A Hong Kong experience. J Affect Disord 2018; 227:643-648.

15. Figueiredo AEB, Silva RM, Mangas RMN, Vieira LJES, Furtado HMJ, Gutierrez DMD, Sousa GS. Impacto do suicídio da pessoa idosa em suas famílias. Cien Saude Colet 2012; 17(8):1993-2002.

16. Asare-Doku W, Osafo J, Akotia CS. The experiences of attempt survivor families and how they cope after a suicide attempt in Ghana: a qualitative study. $B M C$ Psychiatry 2017; 17:178.

17. Pitman AL, Osborn DPJ, Rantell K, King MB. The stigma perceived by people bereaved by suicide and other sudden deaths: A cross-sectional UK study of 3432 bereaved adults. J Psychosom Res 2016; 87;22-29. 
18. Niederkrotenthaler T, Reidenberg DJ, Till B, Gould MS. Increasing help-seeking and referrals for individuals at risk for suicide by decreasing stigma. The role of mass media. Am J Prev Med 2014; 47(3S2):S235-S243.

19. Carmichael V, Whitley R. Suicide portrayal in the Canadian media: examining newspaper coverage of the popular Netflix series '13 Reasons Why'. BMC Public Health 2018; 18:1086.

20. McTernan N, Spillane A, Cully G, Cusack E, O’Reilly T, Arensman E. Media reporting of suicide and adherence to media guidelines. Int J Soc Psychiatry 2018; 64(6):536-544.

21. Chun J, Kim J, Lee S. Fidelity assessment of the suicide reporting guidelines in Korean newspapers. $\mathrm{BMCPu}$ blic Health 2018; 18:1115.

22. Notredame CE, Grandgenèvre P, Pauwels N, Morgiève $M$, Wathelet M, Vaiva G, Séguin M. Leveraging the web and social media to promote access to care among suicidal individuals. Front Psychol 2018; 9:1338.

23. Daine K, Hawton K, Singaravelu V, Stewart A, Simkin S, Montgomery P. The power of the web: a systematic review of studies of the influence of the internet on self-harm and suicide in young people. Plos One 2013; 8:10.

24. Durkee T, Hadlaczky G, Westerlund M, Carli V. Internet pathways in suicidality: a review of the evidence. Int J Environ Res Public Health 2011; 8:3938-3952.

25. National Institute for Mental Health in England (NIMHE). Sensitive Coverage Saves Lives. Leeds: NIMHE; 2007.

26. Robinson J, Bailey E, Hetrick S, Paix S, O’Donnell M, Cox G, Ftanou M, Skehan J. Developing social media-based suicide prevention messages in partnership with young people: exploratory study. JMIR Ment Health 2017; 4(4):e40.

27. Schlichthorst M, King K, Turnure J, Sukunesan S, Phelps A, Pirkis J. Influencing the conversation about masculinity and suicide: evaluation of the Man Up multimedia campaign using Twitter data. JMIR Ment Health 2018; 5(1):e14.

Artigo apresentado em 07/11/2018

Aprovado em 09/07/2019

Versão final apresentada em 11/07/2019

Editores-chefes: Romeu Gomes, Antônio Augusto Moura da Silva 